\title{
AMP-Activated Protein Kinase Beta
}

National Cancer Institute

\section{Source}

National Cancer Institute. AMP-Activated Protein Kinase Beta. NCI Thesaurus. Code C107149.

A family of proteins that function as a regulatory beta subunit for the 5'-AMP-activated protein kinase (AMPK) protein complex. AMPK is a heterotrimeric protein kinase complex that plays a key role in regulating cellular energy metabolism and is comprised of a catalytic alpha subunit and non-catalytic beta and gamma subunits. The beta subunit exists as two isoforms, each is encoded by a separate gene. 